\title{
Anterior Lamellar Keratoplasty in Keratoconus
}

\section{Leopoldo Spadea*}

Department of Biotechnology and Medical-Surgical Sciences, "Sapienza" University of Rome, Latina, Italy

*Corresponding author: Leopoldo Spadea, MD, Department of Biotechnology and Medical-Surgical Sciences, "Sapienza" University of Rome, Latina, Italy; Email: leopoldo.spadea@uniroma1.it

Received date: December 09, 2015; Accepted date: December 10, 2015; Published date: December 14, 2015

Citation: Spadea L (2015) Anterior Lamellar Keratoplasty in Keratoconus. J Eye Cataract Surg 1:6. doi: 10.21767/2471-8300.100006

Copyright: (C) 2015 Spadea L. This is an open-access article distributed under the terms of the Creative Commons Attribution License, which permits unrestricted use, distribution, and reproduction in any medium, provided the original author and source are credited.

\section{Editorial}

Keratoconus $(\mathrm{KC})$ is a progressive, bilateral, but usually asymmetrical ecstatic corneal disease, characterized by progressive corneal protrusion and thinning, that leads to corneal surface distortion. The reported incidence in the general population varies from 1.3 to 25 per 100,000 per year across different populations, with a prevalence of 8.8-229 per 100,000 . Onset typically is at puberty with progression of the disease for 10-20 years after which it tends to stabilize. It occurs in all racial groups and equally affects males and females. [1]

Management of keratoconus depends on its severity and the extent of irregular astigmatism. Mild cases are correctable with spectacles and soft toric contact lenses. However, with the more progressive disease, the cornea becomes more irregular and rigid gas permeable lenses are required. In $15-20 \%$ of keratoconic patient's surgery, typically keratoplasty becomes necessary, as a result of contact lens intolerance, corneal scarring and thinning. [2]

Over the past decade, outcomes have accumulated towards new interventions for keratoconus, which promise to slow down disease progression or correct the refractive defect. These interventions include intracorneal ring implantation, photorefractive keratectomy, phakic intraocular lens implantation and corneal collagen crosslinking [3]. Where contact lenses fail, because of poor visual acuity, intolerance or inability to wear contact lenses or non-resolving corneal hydrop, corneal transplantation became the only feasible therapeutic approach [1].

Keratoplasty has developed rapidly in the past 10 years and penetrating keratoplasty ( $\mathrm{PK})$, a procedure consisting of fullthickness replacement of the cornea, has been the dominant procedure for more than half a century. However, in recent years in all those cases in which corneal disease not involving the endothelium, lamellar technique has rapidly replaced penetrating grafts with better clinical results. This technique aims to selectively replace diseased corneal stroma in a way to minimize unnecessary replacement of the unaffected healthy endothelial layer. Thus, by retaining patient's own endothelium, the risk of endothelial rejection, a major cause of graft failure in PK, is almost eliminated and endothelial cell density is preserved. Consequently, there is no need for long-term immunosuppressive therapy with corticosteroids, decreasing the risk of cataract, glaucoma and infection. Furthermore, since anterior lamellar keratoplasty (ALK) is an extra ocular procedure, it lacks the risk associated with open sky surgery, such as expulsive hemorrhage, endophthalmitis and iris / lens damage. An additional advantage of ALK compared to PK is that sutures can be removed earlier, and final visual recovery occurs sooner.

Newer forms of lamellar keratoplasty techniques have emerged in the last decade or so, revolving around the concept of targeted replacement of diseased corneal layers, including ALK with augmented thickness and deep anterior lamellar keratoplasty.

\section{ALK with augmented thickness}

The recent years have brought about a sea change in the field of corneal transplantation with penetrating keratoplasty being phased to newer lamellar keratoplasty techniques. In keratoconus, the aim of surgery is to augment a thin and steep cornea, and this can be achieved by using a thick lamellar donor of normal curvature, thus tectonically strengthening the cornea (which reduces irregular astigmatism and subsequent ectasia) and reducing corneal steepness (and concomitant high myopia). This is achieved by performing more superficial lamellar dissection of the recipient bed and obtaining a thicker donor lamella. Thanks to the advent of new surgical devices such as advanced microkeratome instrumentation, excimer laser, and femtosecond- laser, the results of lamellar techniques have been encouraging, with rapid visual rehabilitation and vastly reduced risk of immune-mediated transplant rejection. [4]

\section{Deep anterior lamellar keratoplasty}

Over the years, several surgical techniques have been studied and performed in order to obtain the achievement of Descemet's membrane and the elimination of recipient tissue as more is possible, but it is not yet clear whether the full stromal removal provides better results than cases in which a small portion of posterior stroma is left in place. However, although the nomenclature is currently confusing, when ALK enables removal of stroma to the deeper layers, approximately three quarters of stroma or more, some surgeons call this pre- 
descemetic DALK, denoting that at least some posterior stromal layers are retained, and bearing of DM is not achieved.

Current surgical techniques of pre-descemetic DALK involve manual or microkeratome-assisted dissection up to near DM. In descemetic DALK, to create a plane of separation between DM and stromal tissue, current surgical techniques involve manual dissection or injection of air, fluid and viscoelastic into deep stroma. Each technique has its own advantages and disadvantages.

\section{Complications}

Despite the aforementioned advantages of ALK, this surgery is technically challenging to perform, has a significant learning curve, and demands greater operating time compared with penetrating keratoplasty. Complications include intraoperative DM perforation, which may necessitate conversion to penetrating keratoplasty, postoperative DM detachment; interface haze and double anterior chamber due to separation of the donor from host Descemet's membrane. [5]

\section{Conclusions}

Surgical management of keratoconus has improved with advances in surgical techniques, instrumentation, and diagnostic evaluation. ALK procedures should be considered prior to PK as it helps preserves healthy host endothelium, reducing risk of endothelial rejection, ensuring long term graft survival. However, more extensive studies with longer follow-up periods are required to better understand the advantages and disadvantages of ALK.

\section{References}

1. Vazirani J, Basu S (2013) Keratoconus: current perspectives. Clin Ophthalmol 7: 2019-2030.

2. Kirkness CM, Ficker LA, Steele AD, Rice NS (1990) The success of penetrating keratoplasty for keratoconus. Eye (Lond) 4 : 673-688.

3. Spadea L, Mencucci R (2011) Corneal collagen cross-linking. Ophthalmology 118: 2520.

4. Spadea L, Gizzi R, Evangelista Conocchia N, Urbano S. Optical pachymetry-guided custom excimer laser-assisted lamellar keratoplasty for the surgical treatment of keratoconus. J Cataract Refract Surg 2012; 38:1559-1567.

5. Bahar I, Kaiserman I, Srinivasan S, Ya-Ping J, Slomovic AR, et al. (2008) Comparison of three different techniques of corneal transplantation for keratoconus. Am J Ophthalmol 146: 905-912. 